\title{
An acoustic emission source localization approach based on time-reversal technology for additive manufacturing
}

\author{
Xiaodi Yang ${ }^{1}$, Jiazhou Zhou ${ }^{1}$, Chunming Gao, ${ }^{1,}$, Ping Zhang ${ }^{1}$, Tingting Liu ${ }^{2}$, Kai Zhang $^{2}$, \\ and Changdong Zhang ${ }^{2}$ \\ ${ }^{1}$ School of Optoelectronic Science and Engineering, University of Electronic Science and \\ Technology of China, Chengdu, 610054, China \\ ${ }^{2}$ School of mechanical engineering, Nanjing University of Technology, Nanjing, 210094, China
}

\begin{abstract}
The metal additive manufacturing process can inevitably lead to a great temperature gradient in the workpiece. Therefore, the thermal stress deformation and defects seriously affect the processing quality. In this paper, an array acoustic probe is designed on the base plate with the consideration of the time reversal technology. Corresponding simulations is implemented, which are designed to verify the ability of detecting and positioning the workpiece stress release acoustic emission signal. The simulation results demonstrate that the proposed method can position and monitor the random acoustic emission.
\end{abstract}

Keywords: Metal additive manufacturing, Stress release, Time-reversal technology, Soleplate acoustic array, Finite element.

\section{Introduction}

The defects originated from the manufacturing process of metal additive will directly affect the density and mechanical properties of the workpiece, leading to the reduced fatigue life [1] and the incompetence of serving the application. Therefore, it is of great research significance on how to detect and locate the internal defects of the workpiece without damaging its integrity and service performance.

Deformation, crack and other defects are acoustic emission phenomena, which can be detected by acoustic emission detection technology. The traditional acoustic emission location method is not suitable for locating the acoustic emission sources in complex structures. In this paper, hence we introduce the time reversal method into acoustic emission technology, and systematical technical researches on defect detection and location during metal additive manufacturing have been carried out.

The time reversal method can effectively compensate the acoustic path difference and waveform distortion, and realize the adaptive focusing of multi-path acoustic waves [3], it is thus widely used for target reconstruction and positioning in many fields. In 2002,

${ }^{*}$ Corresponding author: gaocm@uestc.edu.cn 
Wang Chenghao et al. [4] published a paper in which they described the time reversal method of conversion reception to realize the identification of ultrasonic target detection with interface. Sheng $x$ et al. [5] verified the feasibility of detecting and locating underwater targets with passive time reversal mirror through experiments, and proposed an improved algorithm to effectively suppress pseudo focusing in 2010. Five years later, Silvano cruciani et al. [6] realized the positioning of medical implants in biological tissues based on pseudo spectral time domain time reversal method. In the year of 2017, the E-TR$\mathrm{RC}$ beamformer designed by MD Hossain et al. [7] improved the positioning of targets in continuous random media. Liao P et al. [8] used XFDTD simulation software to study the influence of electromagnetic signal characteristics in reverberation metal cavity on time anti focusing in 2019. In 2020, Feng x y et al. [9] introduced the concept of entropy into the time reversal process, realizing the reconstruction of electromagnetic source in time and space.

\section{Method, model and positioning simulation}

\subsection{Basic principle of time reversal method}

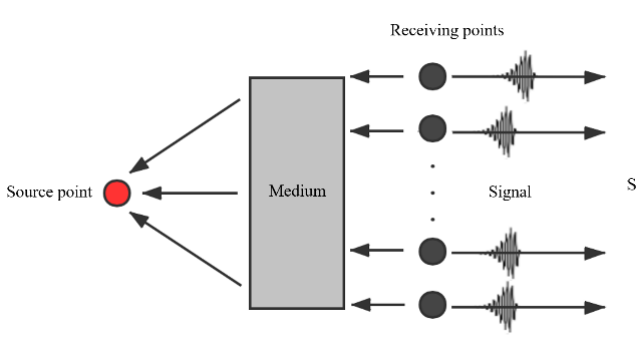

(a)

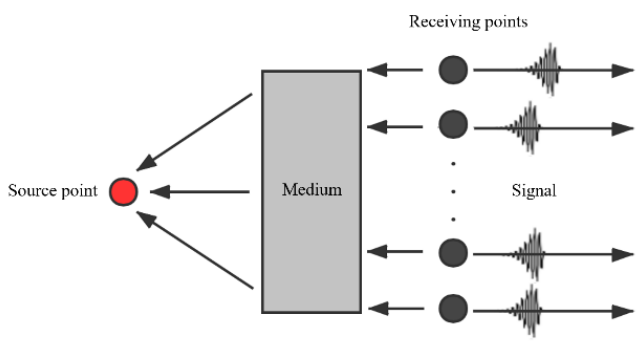

(b)

Fig. 1. Basic principles of time reversal method.

Assuming that there is an acoustic emission source $X(\omega)$ in the space and the transfer function between the acoustic emission source and the sensor $i$ is $H_{i}(\omega)$. Intercepting the $\left[0, t_{c}\right]$ segment of the acoustic emission signal received by each sensor, where $t_{c}$ representing the time delay and each sensor has received the acoustic emission signal at $t_{c}$. After time reversal and reloading to the corresponding sensor, the signal will be emitted again, then the response at the acoustic emission source is:

$$
\begin{aligned}
Z(\omega) & =\left(\sum_{i=1}^{n} H_{i}^{*}(\omega) H_{i}(\omega)\right) e^{j \omega t_{c}} X^{*}(\omega) \\
& =\lambda e^{j \omega t_{c}} X^{*}(\omega)
\end{aligned}
$$

Through the above formula, it can be found that at the acoustic emission source, each mode of the transfer function is in the same direction, and the response is the coherent superposition of the inverse signal, where $n$ represents the number of channel and $\lambda$ is the gain coefficient. At the non-acoustic emission source, the cancellation phenomenon occurs, so the time inverse method focuses in the source point in space. When transforming $Z(\omega)$ to the time domain, we can get: 


$$
\mathrm{z}(t)=\lambda x\left(t_{c}-t\right)
$$

If the acoustic emission source $x(t)$ reaches the peak at time $t_{0}$, then $z(t)$ will reach the peak at time $t_{c}-t_{0}$, realizing the time focusing of the inverse method.

\subsection{Simulation flow of positioning experiment}

The transient pressure acoustic finite element model is created by COMSOL software. The geometry design of the model is shown in figure 2 , in which a point source is added at a random position in the metal structure to simulate the crack source and eight-point sources are evenly distributed in the base plate to simulate the sensor array element. The printing room is a gas environment, so we can set the outer boundary of the model as the impedance boundary. The simulation process is as follows:

(1) Load the sinusoidal signal by the gamma distribution function at the crack source and transmit it. Then the characteristics of modulated signal $x(t)$ are closer to the real acoustic emission signal, which can be expressed as:

$$
x(t)=\lambda t^{\alpha} e^{-f t} \sin (2 \pi f t)
$$

where $\alpha=2, \lambda$ is the normalization coefficient and $f$ is the carrier frequency.

(2) Time inverse normalize the acoustic emission signals received by each array element.

(3) Send the inverse signal to its corresponding array element and transmitting it synchronously when each channel is loaded.

(4) Obtain the maximum instantaneous sound pressure of each point in the metal structure space in the time domain and then descend it to get the global maximum instantaneous sound pressure point.

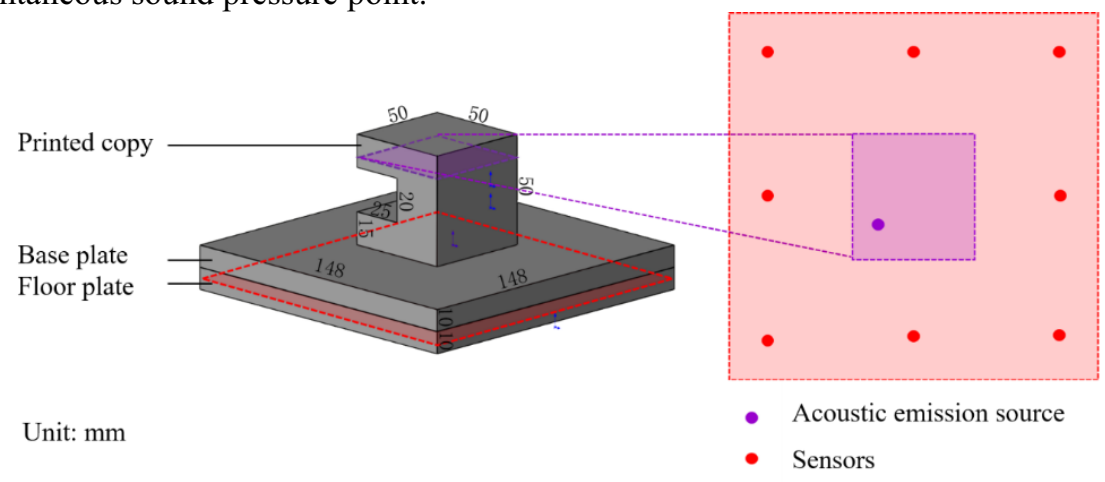

Fig. 2. Basic principles of time reversal method.

\section{Simulation and verification}

The contact surface between metal structure and base plate is $Z=0 \mathrm{~mm}$. Setting the material of metal structure and substrate as TC4, the sound velocity as $5.944 \mathrm{~km} / \mathrm{s}$, the plate material as aluminum alloy 6061 , and the sound velocity as $6.4 \mathrm{~km} / \mathrm{s}$. Fixing the coordinates of crack source as $(-15,-10,40) \mathrm{mm}$, and the coordinates of eight array elements as $(-55,-$ $55,-15),(-55,55,-15),(0,55,-15),(0,55,-15),(0,-55,-15),(55,55,-15),(55,0$, - 
$15),(55,-55,-15) \mathrm{mm}$. The printing room is filled with argon. The impedance value of the impedance boundary is set as $1.784 \mathrm{~kg} / \mathrm{m} 3 \times 323 \mathrm{~m} / \mathrm{s}$ and the carrier frequency of acoustic emission signal is $50 \mathrm{KHz}$.

Table 1. Sorting statistics table of the maximum instantaneous sound pressure(TOP5).

\begin{tabular}{cccc}
\hline $\boldsymbol{X}(\mathbf{m m})$ & $\boldsymbol{Y}(\mathbf{m m})$ & $\boldsymbol{Z}(\mathbf{m m})$ & $\boldsymbol{z}$ \\
\hline-15.0000 & -10.0000 & 40.0000 & 1 \\
-13.7450 & -13.6712 & 41.3802 & 0.9392 \\
-17.2387 & -11.9292 & 40.6139 & 0.9390 \\
-15.9370 & -10.0064 & 45.0000 & 0.9030 \\
-10.6540 & -10.0255 & 41.2500 & 0.8802 \\
\hline
\end{tabular}

The maximum instantaneous sound pressure of each point is obtained during the simulation experiment process and the ranking statistics is shown in table 1 . We can find out that the coordinates of the global maximum instantaneous sound pressure point (positioning point) are $(-15,-10,40) \mathrm{mm}$, which is consistent with the position of the initial crack source. Besides, other high value points are also in the neighborhood range of the positioning point, which proves the theoretical feasibility of time reversal method for locating crack defects.

Figure 3 displays the three-dimensional sound pressure section of the positioning point, from which we can figure out the Obvious focusing phenomenon. At other observation points, the sound pressure value decreases significantly with the change of normal wave order, and the sound pressure value is basically zero, so most areas in the figure 3 are displayed in blue.

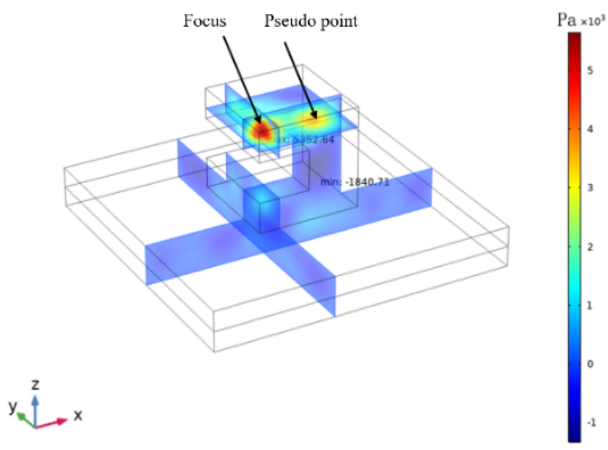

Fig. 3. Anchor point three-coordinate cross-section diagram.

\subsection{Enhancement algorithm}

During the simulation, the positioning accuracy can be affected by not only the high-value points near the positioning point but also the pseudo focus generated under the influence of the same direction interference of the time inverse signal. Therefore, this paper improves the algorithm proposed by $X$ Sheng et al. [5], which is as follows:

(1) The inverse signal on the array element $i$ is transmitted separately to obtain the sound pressure distribution $z_{i}(t)$.

(2) Select $r$ sound pressure distributions for summation, and then multiply the summation results of all combinations. The cumulative sum value $z(t)$ of sound pressure 
is replaced by the cumulative value $z^{\prime}(t)$ as the basis for judging the position of sound source.

$$
\mathrm{z}^{\prime}(t)=\prod_{i=1}^{C_{n}^{r}} \sum_{j \text { in set[i] }} z_{i}(t) \quad \mathrm{r} \in[n / 2, \mathrm{n})
$$

where $C_{n}^{r}$ represents the number of combinations, and set[.] represents the set of combination sequences.

In order to visually display the effect of the improved treatment, the $Z$ coordinate is replaced by the maximum value of the sound pressure accumulated sum value $z(t)$ (accumulated value $z^{\prime}(t)$ ) in the $z$-axis direction to obtain the maximum value distribution map. When $R$ is 7, the results before and after enhancement are shown in figure 4 (a), (b).

Comparing the two figures, it can be seen that after replacing the accumulated value with the sound pressure product value, the original high-value pseudo points are suppressed, the low-value pseudo points basically disappear, and the high-value pseudo points and the real source points can be clearly distinguished, which proves that the improved method has good anti isotropic interference ability. $z^{\prime}(t)$ processing effect is similar to spatial filtering, which suppresses the pseudo point gain and enhances the focusing effect at the sound source.

When the $R$ value is between $N / 2$ and n-1, the smaller the $R$ value is, the more obvious the filtering effect is, and the algorithm can correct the positioning offset to a certain extent.

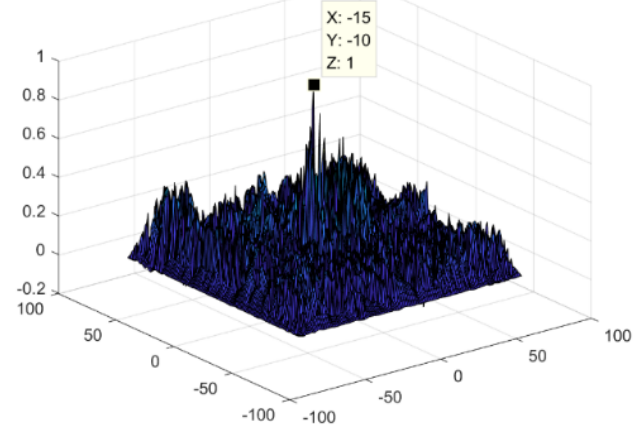

(a) Distribution of the maximum cumulative sum value of sound pressure

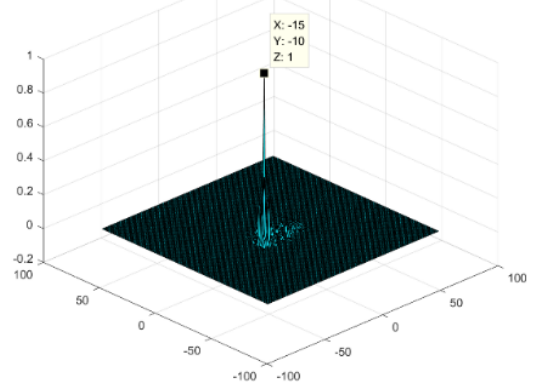

(b) Distribution of the maximum cumulative value of sound pressure

Fig. 4. The maximum value distribution map of sound pressure.

\subsection{Study on influencing factors of time reversal positioning}

\subsubsection{Influence of array element density on time reversal positioning}

After abstracting the bottom plate as a two-dimensional plane and controlling the number of array elements (sensors), we can obtain the anti-focusing results at different times. The comparative analysis of the results demonstrate the influence of array element density on time anti positioning. 
The base plate is made of aluminum alloy 6061 , the sound velocity is set to $5260 \mathrm{~m} / \mathrm{s}$, and the boundary is the impedance boundary. Assuming the sound source is at the position of $(20,10) \mathrm{mm}$ and the carrier frequency is $200 \mathrm{kHz}$. The number of array elements is set to 4 (the first group of sensors), 8 (the first and second groups of sensors) and 16 (the first, second and third groups of sensors), as shown in figure 5 .

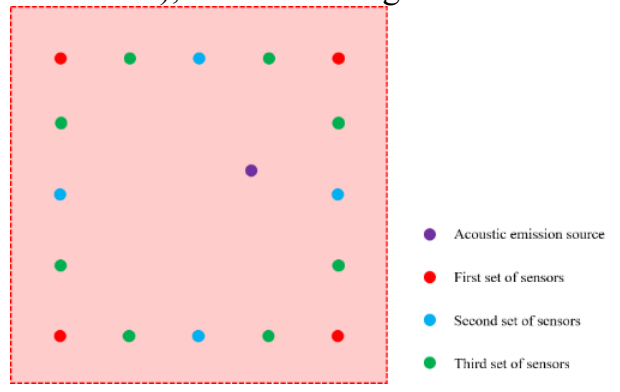

Fig. 5. Sensor and sound source distribution.

As can be seen from figure 6, as the number of array elements increases, the radius of focusing peak basically remains unchanged, while the sound pressure of focusing point gradually increases and the positioning error gradually decreases, indicating that the change of array element density has little effect on the width of main lobe, but can improve the gain and positioning accuracy of main lobe. In addition, it can be seen from the sound field distribution diagram that when the array elements are sparse, the peak values of the main lobe and side lobe are similar, which is easy to confuse the positioning judgment. When the number of array elements is 8 , the difference of the peak values of the main lobe and side lobe is obvious, and the relative difference changes little after further increasing the number of array elements.

When it comes to the real scene, it's difficult for each array element to focus at the same position. However, when the number of array elements is large enough, the relative accuracy of positioning can be guaranteed even if there are some array elements not focusing at the sound source.
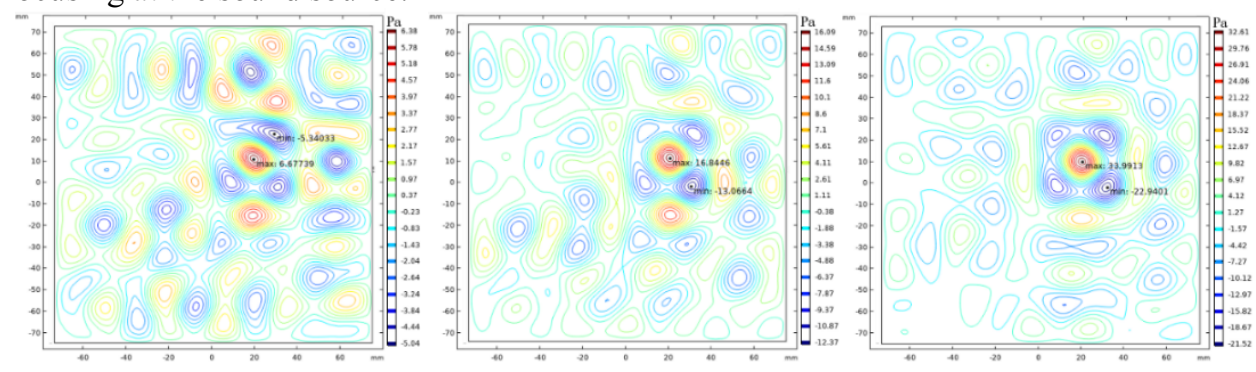

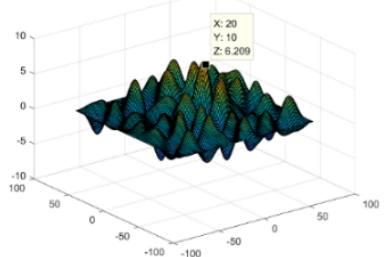

(a)

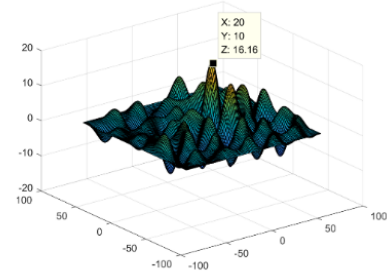

(b)

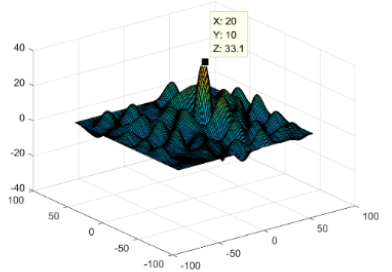

(c)

Fig. 6. Sound field contour map and sound field distribution map at the time of reverse focusing: (a) 4 array elements, positioning coordinates $(18.788,11.729) \mathrm{mm}$ (b) 8 array elements, positioning coordinates $(19.896,11.398) \mathrm{mm}$ (c) 16 array elements, positioning coordinates $(19.394,10.865) \mathrm{mm}$. 


\subsubsection{Influence of carrier frequency on time-domain reverse positioning}

The model is consistent with the model of exploring array element density where the 8array element mode is adopted. Different anti focusing results are obtained by controlling the frequency change of sound source signal. Through the comparative analysis of the results, we analysis the influence of carrier frequency on time anti positioning where carrier frequencies are $100 \mathrm{kHz}, 200 \mathrm{kHz}$ and $300 \mathrm{kHz}$ respectively.
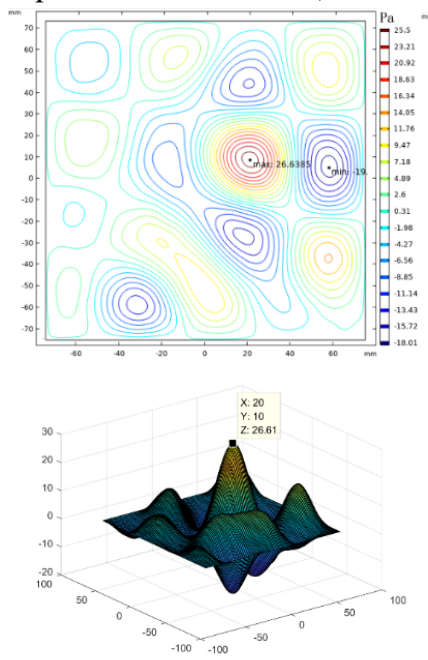

(a)
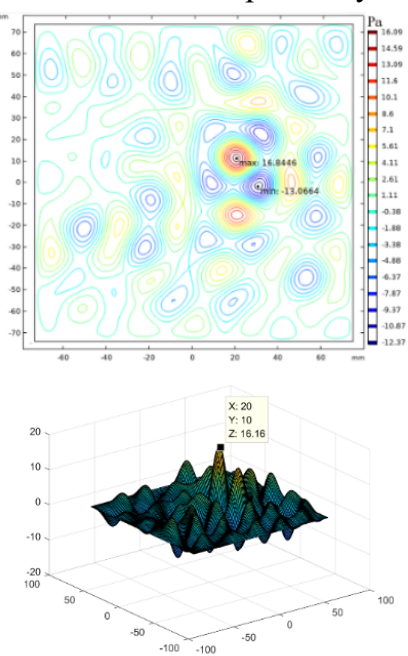

(b)
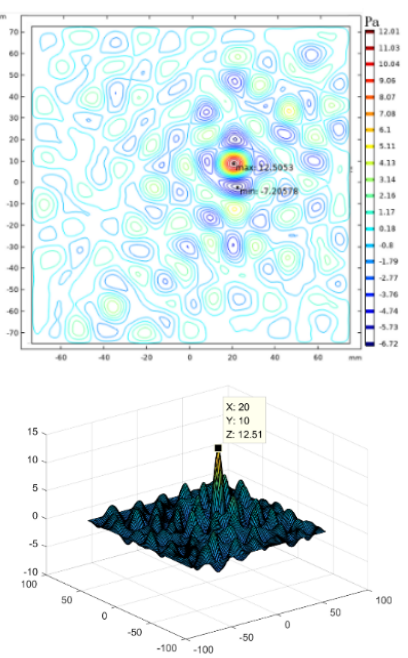

(c)

Fig. 7. Sound field contour map and sound field distribution map at the time of reverse focusing: (a) $100 \mathrm{KHz}$, positioning coordinates $(20.672,9.4711) \mathrm{mm}$ (b) $200 \mathrm{KHz}$, positioning coordinates $(19.896$, $11.398) \mathrm{mm}$ (c) $300 \mathrm{KHz}$, positioning coordinates $(20,10) \mathrm{mm}$.

As we known, the larger the carrier frequency, the faster the phase change. Therefore, it can be seen in figure 7 that the focus peak gradually becomes sharp with the increase of carrier frequency, but the peak gradually decreases due to signal attenuation. Due to the drastic change of phase, the distribution of sound field fluctuates greatly, forming more pseudo focuses, which reduces the positioning stability. Besides, from the change of positioning coordinates, it can be concluded that the larger the carrier frequency is, the more accurate the positioning is.

\subsubsection{Influence of sound source spacing on time reversal location}

Considering the simultaneous generation of cracks and the simultaneous emission of two sound sources, different anti focusing results are obtained by controlling the sound source spacing. Through the comparative analysis of the results, we can draw the conclusion on the influence of sound source spacing for time anti positioning.

The basic model remains unchanged. The 8-element mode is adopted. Two-point sources are placed on the bisector of the first and third quadrants. The two-point sources are symmetrical centered on the origin. The point sources are sinusoidal signals with five cycles set to the frequency of $0.5 \mathrm{MHz}$, and the spacing $D$ is $10 \sqrt{2}, 20 \sqrt{2}$ 和 $40 \sqrt{2} \mathrm{~mm}$ respectively.

As shown in figure 8 , when $D=10 \sqrt{2} \mathrm{~mm}$, the two anti-converging focusing peaks are mixed and become difficult to distinguish. With the increase of $D$, the side lobe decreases, the position of focusing peak becomes clear, and the peak decreases. It is natural to 
consider that the limit resolution is directly related to the half width of the time anti focusing peak and the smaller the half width, the higher the resolution.

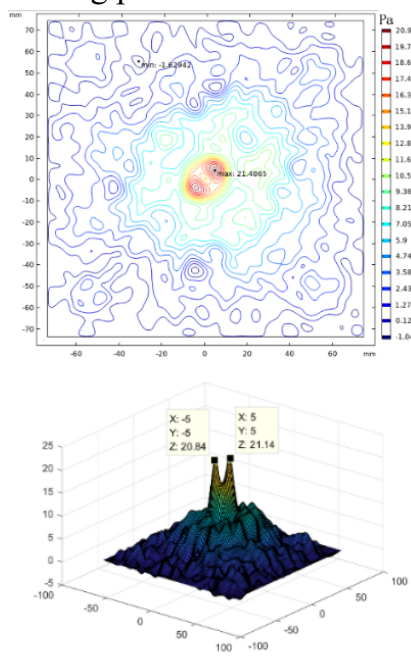

(a) $\mathrm{D}=10 \sqrt{2} \mathrm{~mm}$
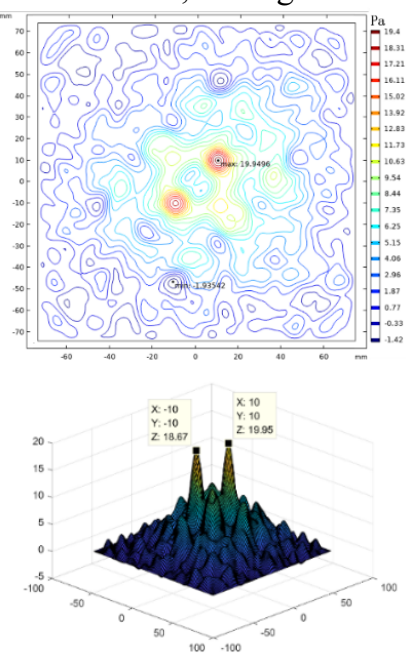

(b) $\mathrm{D}=20 \sqrt{2} \mathrm{~mm}$
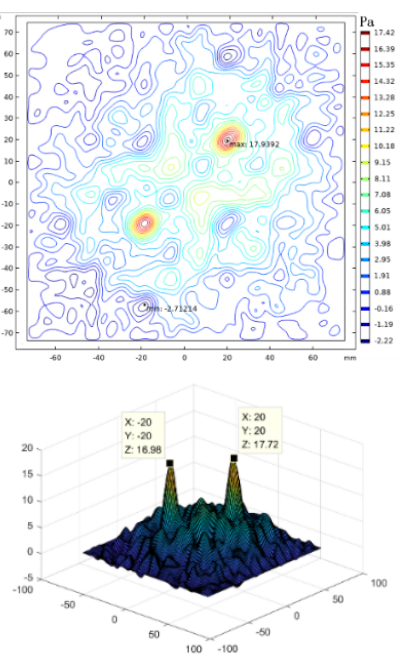

(c) $\mathrm{D}=40 \sqrt{2} \mathrm{~mm}$

Fig. 8. Sound field contour map and sound field distribution map at the time of reverse focusing.

\subsubsection{Influence of floor structure on time reverse positioning.}

In the three-dimensional simulation experiment, the geometric structure of the bottom plate is a standard cuboid, which can't be embedded into the sensor circuit and has no practical application ability. Therefore, the bottom plate structure is modified in this paper, and a back groove is opened on the back of the original structure to embed the sensor circuit, as shown in figure 9. Slot structure increases the complexity of sound propagation and will inevitably have a negative impact on positioning. Because of it, this section mainly explores the impact of bottom plate structure on time anti positioning.

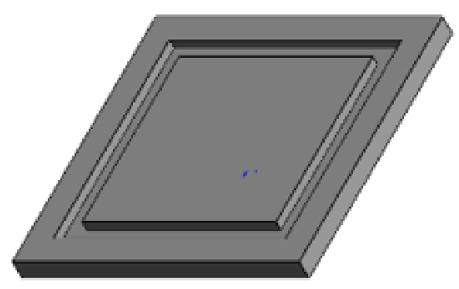

Fig. 8. Floor structure with return groove.

We keep the basic settings of the simulation model unchanged but replace the bottom plate with a grooved bottom plate. The groove depth is $5 \mathrm{~mm}$ and the array elements are distributed on the bottom surface of the groove. After the simulation, the maximum value of global instantaneous sound pressure is $(-9.6490,-4.6730,42.500) \mathrm{mm}$, with large positioning error. It can be seen from figure 9 (a) that the focus divergence and focus peak are low, which is three orders of magnitude smaller than the previous simulation sound pressure value. This is caused by the interface reflection of the time back signal at the slot structure. 
In order to eliminate the negative impact of the structure, after embedding the sensor, we design to fill the return groove with rubber to make the acoustic characteristic impedance of the upper and lower materials of the array element similar, so as to reduce the reflection and scattering of sound and thus decrease the influence of multipath effect. The rubber sound velocity is set to $1.5 \mathrm{~km} / \mathrm{s}$, and the positioning point and the initial point source position coincide after the filling of the rubber. It can be seen from figure 9 (b) that the focusing effect is obvious, and the focusing peak value is restored to the same order of magnitude when using the non-grooved bottom plate.

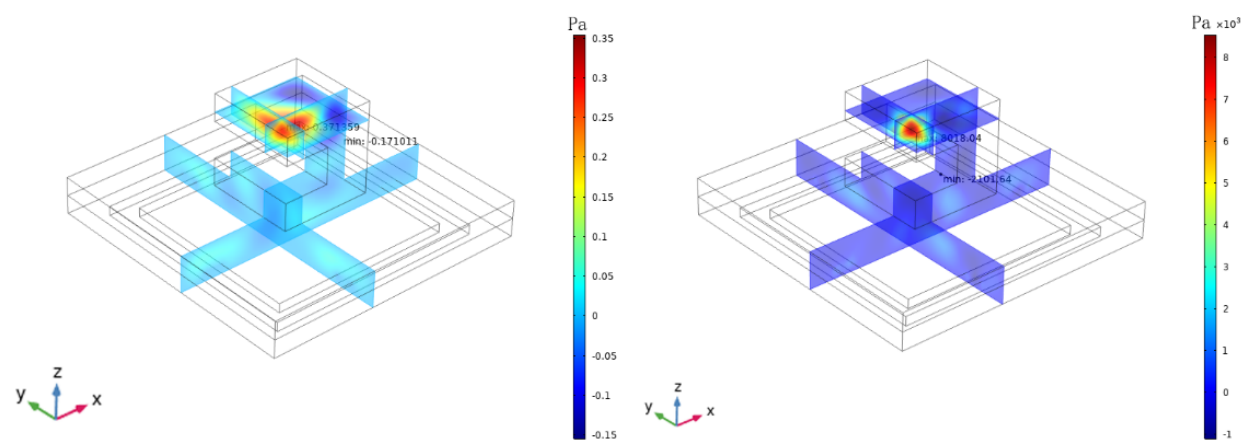

(a) Recirculation groove

(b) Recirculation groove filled with rubber

Fig. 9. Three-section sound pressure distribution diagram of point source coordinates.

\subsection{Simulation positioning error}

In the experiment of exploring the influencing factors of time anti positioning, the qualitative analysis is conducted for exploring the influence of various factors on positioning. In this section, we selected the filled rubber base plate and assumed to ignore the multi-source scenario. The quantitative analysis is mainly made to investigate the influence of element density and carrier frequency on time reverse positioning in order to determine the average positioning error under various conditions.

There are 21 point-sources in the U-shaped structural member as the sampling points for the calculation of average positioning error (see figure 10), and the initial state of all point sources is disabled. We can see that the point source and model structure are mirror symmetrical in the $\mathrm{x}=0$ plane, but the meshing is not completely symmetrical, so the positioning results of mirror symmetrical point pairs do not have symmetry.

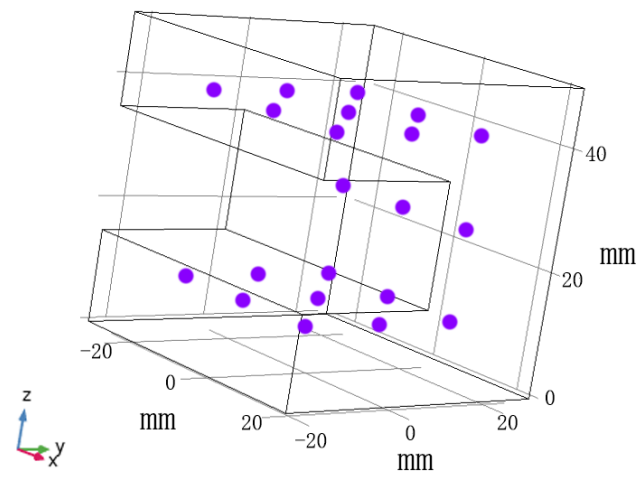

Fig. 10. Sampling point distribution map. 
The carrier frequencies are $25 \mathrm{kHz}, 50 \mathrm{KHz}$ and $100 \mathrm{kHz}$ respectively. After the carrier frequency is fixed, one of the point sources is activated. When the acoustic signal is received by all receiving points, 4,8 and 16 array elements are triggered in turn for time back transmission and the positioning coordinates were recorded. Each point source needs to be transmitted separately, that is, 21 positioning coordinates will be obtained in the combination of each carrier frequency and array element density. After calculating the average value of the Euclidean distance between the positioning coordinates and the real point source position, we can obtain the average positioning error under every combination condition. See table 2 for the statistical results.

Table 2. Statistical table of simulation positioning average error (unit: $\mathrm{mm}$ ).

\begin{tabular}{|c|c|c|c|}
\hline \multirow[b]{2}{*}{$\begin{array}{c}\text { Carrier } \\
\text { frequency }\end{array}$} & \multicolumn{3}{|c|}{ Number of elements } \\
\hline & 4 & 8 & 16 \\
\hline $25 \mathrm{KHz}$ & 2.8981 & 2.8022 & 1.5385 \\
\hline $50 \mathrm{KHz}$ & 2.6589 & 2.7231 & 1.3969 \\
\hline $100 \mathrm{KHz}$ & 2.3989 & 2.1883 & 0.9320 \\
\hline
\end{tabular}

The grid size represents the spatial resolution. In order to remain the spatial resolution unchanged, a unified grid division standard is adopted. The results of the statistical table are consistent with the expectation. The positioning error decreases with the increase of carrier frequency and the number of array elements. Furthermore, in the process of positioning error statistics, it is found that in this model, the farther the sound source is away from the interface, the smaller the positioning error is, and the positioning point close to the interface will shift away from the interface.

\section{Conclusion}

In this paper, a method to locate the position of acoustic emission signal, which is based on the principle of time reversal and the distributed acoustic sensor array on the base plate, is established. Simulation on finite element software verified the feasibility of this method. Simulation results demonstrate that (1) The growth of the number of array elements and the increase of carrier frequency will reduce the positioning error and improve the positioning accuracy. (2) With the increase of the number of array elements, the peak difference between focus and pseudo focus gradually grows, which is convenient for positioning judgment. (3) As the carrier rises, the pseudo focus and the positioning stability declines. (4) Due to the refletion of the interface, the positioning result of the sound source close to the boundary will shift away from the interface.

This work was supported by the National KeyResearch and Development Program of China (Grant Nos. 2017YFB-1103002).

\section{References}

1. Shi, Y. W., Yang, P. H., Liang, J., \& Wang, Z. (2016, June). Relations among ultrasonic testing results and defect characteristics and material properties of laser additive manufacturing titanium alloy. In Proceedings of the 19th World Conference of Non-Destructive Testing, Munich, Germany (pp. 13-17).

2. Yang, R. F. , \& Ma, T. H. . (2006). A study on the applications of acoustic emission technique. Journal of North University of China(Natural Science Edition).27(05):456461. 
3. Huiying, M. A. , \& Zeng, X. . (2015). Time reversal method and applications for acoustic localization. Audio Engineering. (07), 30-34.

4. Wang, C. Y., \& Wei, W..(2002). Distinguishing between target and interface in ultrasonic detection by a modified time reversal method. Chinese Journal of Acoustics, 27(3), 193-197

5. Sheng, X., Luo, F., Mei, J., \& Guo, Y. (2010, September). Target passive location by time reversal mirror using vertical array. In OCEANS 2010 MTS/IEEE SEATTLE (pp. 1-5). IEEE.

6. Cruciani, S., \& Feliziani, M. (2015). UWB source localization by using the pseudospectral time-domain time-reversal method in biological tissues. IEEE Transactions on Magnetics, 51(3), 1-4.

7. Hossain, M. D., \& Mohan, A. S. (2017). Eigenspace time-reversal robust Capon beamforming for target localization in continuous random media. IEEE antennas and wireless propagation letters, 16, 1605-1608.

8. Liao, P., Hu, B. J., Lin, Z. W., Wen, Q., \& Zheng, L. L. (2019, May). Effect of signal characteristics on focusing property of time reversal electromagnetic wave. In 2019 International Conference on Microwave and Millimeter Wave Technology (ICMMT) (pp. 1-3). IEEE.

9. Feng, X. Y., Chen, Z. D., \& Liang, J. C. (2020, August). The entropy technique for the time-reversal source reconstruction. In 2020 IEEE/MTT-S International Microwave Symposium (IMS) (pp. 158-160). IEEE.

10. Dorme, C., \& Fink, M. (1995). Focusing in transmit-receive mode through inhomogeneous media: The time reversal matched filter approach. The Journal of the Acoustical Society of America, 98(2), 1155-1162. 